\title{
The Precise Inner Solutions of Gravity Field Equations of Hollow and Solid Spheres and the Theorem of Singularity
}

\author{
Xiaochu Mei \\ Institute of Innovative Physics in Fuzhou, Department of Physics, Fuzhou University, Fuzhou, China \\ E-mail: ycwlyjs@yeah.net \\ Received May 26, 2011; revised June 28, 2011; accepted July 12, 2011
}

\begin{abstract}
The precise inner solutions of gravity field equations of hollow and solid spheres are calculated in this paper. To avoid space curvature infinite at the center of solid sphere, we set an integral constant to be zero directly at present. However, according to the theory of differential equation, the integral constant should be determined by the known boundary conditions of spherical surface, in stead of the metric at the spherical center. By considering that fact that the volumes of three dimensional hollow and solid spheres in curved space are different from that in flat space, the integral constants are proved to be nonzero. The results indicate that no matter what the masses and densities of hollow sphere and solid sphere are, there exist space-time singularities at the centers of hollow sphere and solid spheres. Meanwhile, the intensity of pressure at the center point of solid sphere can not be infinite. That is to say, the material can not collapse towards the center of so-called black hole. At the center and its neighboring region of solid sphere, pressure intensities become negative values. There may be a region for hollow sphere in which pressure intensities may become negative values too. The common hollow and solid spheres in daily live can not have such impenetrable characteristics. The results only indicate that the singularity black holes predicated by general relativity are caused by the descriptive method of curved space-time actually. If black holes exist really in the universe, they can only be the Newtonian black holes, not the Einstein's black holes. The results revealed in the paper are consistent with the Hawking theorem of singularity actually. They can be considered as the practical examples of the theorem.
\end{abstract}

Keywords: General Relativity, Inner Solutions of Hollow and Solid Spheres, Black Hole, Theorem of Singularity

\section{Introduction}

We know that the static solutions of the Einstein's equation of gravity field with spherical symmetry are the Schwarzschild solutions which include inner and external ones. We consider a static and uniform sphere with radius $r_{0}$ and constant density $\rho_{0}$, inner pressure intensity $p(r)$ is related to coordinate but does not depends on time. By considering static energy momentum tensor of idea fluid, the Schwarzschild inner solution is $[1,2]$.

$$
\begin{aligned}
\mathrm{d} s^{2}= & c^{2}\left(\frac{3}{2} \sqrt{1-r_{0}^{2} / R^{2}}-\frac{1}{2} \sqrt{1-r^{2} / R^{2}}\right)^{2} \mathrm{~d} t^{2} \\
& -\left(1-\frac{r^{2}}{R^{2}}\right)^{-1} \mathrm{~d} r^{2}-r^{2}\left(\mathrm{~d} \theta^{2}+\sin ^{2} \theta \mathrm{d} \phi^{2}\right)
\end{aligned}
$$

Here $R^{2}=3 c^{2} / 8 \pi G \rho_{0}$. The metric is finite at the center point of sphere. However, it should be pointed out that in the process of solving the Einstein's equation of gravity field, what we obtain is actually

$$
g_{11}(r)=\left(1-\frac{r^{2}}{R^{2}}+\frac{A}{r}\right)^{-1}
$$

It can be proved based on (2) that the space curvature is infinite at point $r=0$. In order to avoid the infinity, we let integral constant $A$ to be zero directly in the current theory. However, according to the theory of differential equation, integral constant should be determined by the known boundary conditions on spherical surface, in stead of the metric at the spherical center which is unknown. By considering the fact that the volume of sphere in curved space is different from that in flat space, 
we can prove $A \neq 0$. Therefore, no matter what the mass and density of solid sphere are, the curvature infinity at the center of sphere is inevitable.

On the other hand, according to the current theory, the inner pressure intensity of sphere is [3].

$$
p(r)=\rho_{0} c^{2} \frac{\sqrt{1-(r / R)^{2}}-\sqrt{1-\left(r_{0} / R\right)^{2}}}{3 \sqrt{1-\left(r_{0} / R\right)^{2}}-\sqrt{1-(r / R)^{2}}}
$$

On the spherical surface $r=r_{0}$ we have $p\left(r_{0}\right)=0$. To make pressure intensity to be finite at the center of sphere, we have to introduce a constraint condition for spherical radius with

$$
r_{0}^{2}<\frac{8 R^{2}}{9} \quad \text { or } \quad r_{0}>\frac{9 r_{g}}{8}
$$

Here $r_{g}=2 G M / c^{2}$ is the Schwarzschild radius. If $r_{0} \leq 9 r_{g} / 8$, pressure intensity will become infinite. In this case, stable solution is impossible and material would collapse towards the center of sphere so that singularity black holes appear. However, if integral constant $A \neq 0$, pressure intensity (3) and constraint condition (4) will be changed. All calculations based on (3) and (4) about high density celestial bodies in the current astrophysics should be reconsidered.

Let's first strictly calculate the solutions of gravity field equations of hollow and solid spheres, and then discuss the problems of singularities below.

\section{The Strict Inner Solution of Gravity Field of Hollow Sphere}

Suppose that the inner radius of hollow sphere is $R_{1}$ and the external radius is $R_{2}$, the gravity mass is $M$. The region $I_{3}$ with $r>R_{2}$ and the region $I_{1}$ with $r<R_{1}$ are vacuum. The region $I_{2}$ inside two spherical shells with $R_{1}<r<R_{2}$ is composed of complete liquid with constant density $\rho_{0}$ and pressure intensity $p(r)$. Because material is distributed with spherical symmetry, the metric can be written as

$$
\mathrm{d} s^{2}=c^{2} \mathrm{e}^{v(r)} \mathrm{d} t^{2}-\mathrm{e}^{\lambda(r)} \mathrm{d} r^{2}-r^{2}\left(\mathrm{~d} \theta^{2}+\sin ^{2} \theta \mathrm{d} \phi^{2}\right)
$$

The solution of the Einstein's equation of gravity in the region $I_{3}$ is the well-known Schwarzschild metric with

$$
\begin{gathered}
g_{00}(r)=\mathrm{e}^{v(r)}=\left(1+\frac{A_{3}}{r}\right) \\
g_{11}(r)=-\mathrm{e}^{\lambda(r)}=-\left(1+\frac{A_{3}}{r}\right)^{-1}
\end{gathered}
$$

In order to determine integral constant $A_{3}$, we compare (6) with the Newtonian theory under the asymptotic condition with $r \rightarrow \infty$

$$
\left.g_{00}(r)\right|_{r \rightarrow \infty}=1+\frac{2 \psi(r)}{c^{2}}, \quad \psi(r)=-\frac{G M}{r}
$$

Here $M$ is the static gravity mass of hollow sphere in the Newtonian theory. By comparing (6) with (7), we obtain $A_{3}=-2 G M / c^{2}$. So we have the same result for hollow sphere

$$
g_{00}(r)=\left(1-\frac{2 G M}{c^{2} r}\right), \quad g_{11}(r)=-\left(1-\frac{2 G M}{c^{2} r}\right)^{-1}
$$

To calculate the metric in the region $I_{2}$ beneath two spherical shells, the mixing energy momentum tensor of complete fluid is used [1]

$$
T_{0}^{0}=\rho_{0} c^{2}, T_{1}^{1}=T_{2}^{2}=T_{3}^{3}=-p_{0}, R_{1} \leq r \leq R_{2}
$$

The Einstein's equation of gravity field is

$$
R_{\mu}^{v}-\frac{1}{2} g_{\mu}^{v} R=-\frac{8 \pi G T_{\mu}^{v}}{c^{4}}
$$

According to the standard procedure of calculation in general relativity, we obtain

$$
\begin{gathered}
\mathrm{e}^{-\lambda(r)}\left(\frac{\lambda^{\prime}}{r}-\frac{1}{r^{2}}\right)+\frac{1}{r^{2}}=\frac{8 \pi G \rho_{0} c^{2}}{c^{4}} \\
\mathrm{e}^{-\lambda(r)}\left(\frac{v^{\prime}}{r}+\frac{1}{r^{2}}\right)-\frac{1}{r^{2}}=\frac{8 \pi G p(r)}{c^{4}} \\
\mathrm{e}^{-\lambda(r)}\left(\frac{v^{\prime \prime}}{2}-\frac{\lambda^{\prime} v^{\prime}}{4}+\frac{v^{\prime 2}}{4}+\frac{v^{\prime}-\lambda^{\prime}}{2 r}\right)=\frac{8 \pi G p(r)}{c^{4}}
\end{gathered}
$$

The integral of (11) is

$$
g_{11}(r)=-\mathrm{e}^{\lambda(r)}=-\left(1-\frac{r^{2}}{R^{2}}+\frac{A_{2}}{r}\right)^{-1}
$$

In which $R^{2}=3 c^{2} / 8 \pi \mathrm{G} \rho_{0}$ and $A_{2}$ is an integral constant. We will prove $A_{2} \neq 0$ in the next section. (12) minus (13) then multiplied by $2 / r$ and considering (11), we obtain

$$
\frac{\mathrm{d} p(r)}{\mathrm{d} r}+\left[\rho_{0} c^{2}+p(r)\right] \frac{v^{\prime}(r)}{2}=0
$$

The integral of (15) is

$$
\rho_{0} c^{2}+p(r)=B_{2} \mathrm{e}^{-v(r) / 2}
$$

Here $B_{2}$ is an integral constant. By considering (11), (12) and (16), we obtain

$$
\mathrm{e}^{v(r) / 2} \mathrm{e}^{-\lambda(r)}\left[\frac{\lambda^{\prime}(r)+v^{\prime}(r)}{r}\right]=\frac{c^{4} B_{2}}{4 \pi G}
$$


On the other hand, by taking the differential of (14) with respect to $r$, we get

$$
\lambda^{\prime}(r) \mathrm{e}^{-\lambda(r)}=\frac{2 r}{R^{2}}+\frac{A_{2}}{r^{2}}
$$

Substituting (14) and (18) in (17), we get

$$
\mathrm{e}^{v(r) / 2}\left[\frac{2}{R^{2}}+\frac{A_{2}}{r^{3}}+v^{\prime}(r)\left(\frac{1}{r}-\frac{r}{R^{2}}+\frac{A_{2}}{r^{2}}\right)\right]=\frac{c^{4} B_{2}}{4 \pi G}
$$

By considering the relation $\mathrm{e}^{v(r) / 2} v^{\prime}(r)=2 \mathrm{de}^{v(r) / 2} / \mathrm{d} r$, (19) can be written as

$$
\frac{\mathrm{de}^{v(r) / 2}}{\mathrm{~d} r}+P(r) \mathrm{e}^{v(r) / 2}=B_{2} K(r)
$$

here

$$
\begin{aligned}
& P(r)=\frac{1}{2 r}-\frac{1-3 r^{3} / R^{2}}{2\left(A_{2}+r-r^{3} / R^{2}\right)} \\
& K(r)=\frac{c^{4} r^{2}}{8 \pi G\left(A_{2}+r-r^{3} / R^{2}\right)}
\end{aligned}
$$

The integral of (20) is

$$
\mathrm{e}^{v(r)}=\left[\mathrm{e}^{-\int P(r) \mathrm{d} r}\left(B_{2} \int \mathrm{e}^{\int P(r) d r} K(r) \mathrm{d} r+C_{2}\right)\right]^{2}
$$

Here $C_{2}$ is an integral constant. If let $A_{2}=0$ in (14) and (21), we reach the result of current theory [1]

$$
\begin{aligned}
& g_{00}(r)=\mathrm{e}^{v(r)}=\left(B-C \sqrt{1-\frac{r^{2}}{R^{2}}}\right)^{2} \\
& g_{11}(r)=-\mathrm{e}^{\lambda(r)}=-\left(1-\frac{r^{2}}{R^{2}}\right)^{-1}
\end{aligned}
$$

In which constants $B=c^{4} B_{2} R^{2} /(8 \pi G), C=-C_{2}$. If $A_{2} \neq 0$, we have

$$
\mathrm{e}^{-\int P(r) \mathrm{d} r}=\mathrm{e}^{-\frac{\ln r}{2}} \mathrm{e}^{\frac{1}{2} \int Q(r) \mathrm{d} r}=\frac{1}{\sqrt{r}} \mathrm{e}^{-\frac{1}{2} \int Q(r) \mathrm{d} r}
$$

here

$$
Q(r)=\frac{1-3 r^{3} / R^{2}}{A_{2}+r-r^{3} / R^{2}}=3+\frac{1-3 A_{2}-3 r}{A_{2}+r-r^{3} / R^{2}}
$$

let

$$
\frac{1}{A_{2}+r-r^{3} / R^{2}}=\frac{\alpha_{0}}{\left(\alpha_{1}+\alpha_{2} r\right)}+\frac{\alpha_{3}+\alpha_{4} r}{\left(\alpha_{5}+\alpha_{6} r+\alpha_{7} r^{2}\right)}
$$

The forms of constants $\alpha_{i}=\alpha_{i}\left(A_{2}, R\right) \neq 0$ are complex, but it is unnecessary for us to write them out. We have

$$
\begin{aligned}
\int Q(r) \mathrm{d} r= & 3\left(1-\frac{\alpha_{0}}{\alpha_{2}}\right) r \\
& +\left[\frac{\alpha_{0}\left(1-3 A_{2}\right)}{\alpha_{2}}+\frac{3 \alpha_{0} \alpha_{1}}{\alpha_{2}^{2}}\right] \ln \left(\alpha_{1}+\alpha_{2} r\right) \\
& -\frac{3}{2 \alpha_{7}} \ln \left(\alpha_{5}+\alpha_{6} r+\alpha_{7} r^{2}\right)+\frac{1-3 A_{2}+\alpha_{6} /\left(2 \alpha_{7}\right)}{\sqrt{\alpha_{6}^{2}-4 \alpha_{5} \alpha_{7}}} \\
& \times \ln \frac{2 \alpha_{7} r+\alpha_{6}-\sqrt{\alpha_{6}^{2}-4 \alpha_{5} \alpha_{7}}}{2 \alpha_{7} r+\alpha_{6}+\sqrt{\alpha_{6}^{2}-4 \alpha_{5} \alpha_{7}}}
\end{aligned}
$$

we write

$$
\begin{aligned}
& F(r)= \mathrm{e}^{\frac{1}{2} \int Q(r) \mathrm{d} r} \\
&= \mathrm{e}^{\frac{3}{2}\left(1-\alpha_{0} / \alpha_{2}\right) r} \times\left(\alpha_{1}+\alpha_{2} r\right)^{\left[\alpha_{0}\left(1-3 A_{2}\right) / \alpha_{2}+3 \alpha_{0} \alpha_{1} / \alpha_{2}^{2}\right]} \\
& \times\left(\alpha_{5}+\alpha_{6} r+\alpha_{7} r^{2}\right)^{-\frac{3}{2 \alpha_{7}}} \\
& \times\left(\ln \frac{2 \alpha_{7} r+\alpha_{6}-\sqrt{\alpha_{6}^{2}-4 \alpha_{5} \alpha_{7}}}{2 \alpha_{7} r+\alpha_{6}+\sqrt{\alpha_{6}^{2}-4 \alpha_{5} \alpha_{7}}}\right) \underbrace{\frac{1-3 A_{2}+\alpha_{6} /\left(2 \alpha_{7}\right)}{\sqrt{\alpha_{6}^{2}-4 \alpha_{5} \alpha_{7}}}} \\
& D(r)=\int \frac{\sqrt{r} K(r)}{F(r)} \mathrm{d} r
\end{aligned}
$$

let $r=0$ in (28), we get

$$
\begin{aligned}
F(0)= & \left(\alpha_{1}\right)^{\left[\alpha_{0}\left(1-3 A_{2}\right) / \alpha_{2}+3 \alpha_{0} \alpha_{1} / \alpha_{2}^{2}\right]}\left(\alpha_{5}\right)^{-3 /\left(2 \alpha_{7}\right)} \\
& \times\left(\ln \frac{\alpha_{6}-\sqrt{\alpha_{6}^{2}-4 \alpha_{5} \alpha_{7}}}{\alpha_{6}+\sqrt{\alpha_{6}^{2}-4 \alpha_{5} \alpha_{7}}}\right)^{\frac{1-3 A_{2}+\alpha_{6} /\left(2 \alpha_{7}\right)}{\sqrt{\alpha_{6}^{2}-4 \alpha_{5} \alpha_{7}}}} \\
& \neq 0
\end{aligned}
$$

Therefore, $D(r)$ is finite at point $r=0$. We can write (22) as

$$
\mathrm{e}^{v(r)}=\frac{F^{2}(r)}{r}\left[C_{2}+B_{2} D(r)\right]^{2}
$$

In the region $I_{2}$, the metric can be written as at last

$$
\begin{aligned}
\mathrm{d} s^{2}= & \frac{c^{2} F^{2}(r)}{r}\left[C_{2}+B_{2} D(r)\right]^{2} \mathrm{~d} t^{2} \\
& -\left(1-\frac{r^{2}}{R^{2}}+\frac{A_{2}}{r}\right)^{-1} \mathrm{~d} r^{2}-r^{2}\left(\mathrm{~d} \theta^{2}+\sin ^{2} \theta \mathrm{d} \phi^{2}\right)
\end{aligned}
$$

In the vacuum region $I_{1}$ of hollow sphere cavity, the 
solution of the Einstein's equation of gravity field is still the Schwarzschild solution

$$
g_{00}(r)=\left(1+\frac{A_{1}}{r}\right), \quad g_{11}(r)=-\left(1+\frac{A_{1}}{r}\right)^{-1}
$$

Let's determine the integral constants $A_{1}, A_{2}, B_{2}$ and $C_{2}$ below.

\section{The Calculations of Integral Constants for Hollow Sphere}

By considering the continuity of metric tensors on the external spherical surface $r=R_{2}$, according to (8), (14) and (32), we have

$$
\begin{gathered}
A_{2}=-\frac{2 G M}{c^{2}}+\frac{R_{2}^{3}}{R^{2}}=-\frac{2 G M}{c^{2}}+\frac{8 \pi G \rho_{0} R_{2}^{3}}{3 c^{2}} \\
1-\frac{2 G M}{c^{2} R_{2}}=\frac{F^{2}\left(R_{2}\right)}{R_{2}}\left[C_{2}+B_{2} D\left(R_{2}\right)\right]^{2}
\end{gathered}
$$

Similarly, on the internal sphere surface with $r=R_{1}$, according to (14), (33), (35) and (36), we have

$$
\begin{aligned}
& A_{1}=-\frac{2 G M}{c^{2}}+\frac{8 \pi G \rho_{0}}{3 c^{2}}\left(R_{2}^{3}-R_{1}^{3}\right) \\
& 1+\frac{A_{1}}{R_{1}}=\frac{F^{2}\left(R_{1}\right)}{R_{1}}\left[C_{2}+B_{2} D\left(R_{1}\right)\right]^{2}
\end{aligned}
$$

Let's prove $A_{1} \neq 0$ in curved space now. If space is flat, the relation between mass $M$ and volume $V_{0}$ of hollow sphere is $M=\rho_{0} V_{0}$ with

$$
V_{0}=\frac{4 \pi G}{3}\left(R_{2}^{3}-R_{1}^{3}\right)
$$

It should be emphasized that $M$ is the Newtonian gravity mass. We introduce it by considering the asymptotic relation (7) between the Einstein's theory and the Newtonian theory of gravity. Substitute (38) in (37), we get $A_{1}=0$. This is just the current calculating result of general relativity.

However, (38) can not hold in curved space. Because there is a length contraction along the direction of radius, we should have $\mathrm{d} l^{\prime}=\mathrm{d} l / \sqrt{-g_{11}(r)}$ and $\mathrm{d} \sigma^{\prime}=\mathrm{d} \sigma$, so $\mathrm{d} V^{\prime}=\mathrm{d} l^{\prime} d \sigma^{\prime}=\mathrm{d} V / \sqrt{-g_{11}(r)}$. In the curved space, the volume should be calculated by the following formula

$$
V^{\prime}=\int \frac{\mathrm{d} V}{\sqrt{-g_{11}(r)}}=\int_{R_{1}}^{R_{2}} \frac{4 \pi r^{2} \mathrm{~d} r}{\sqrt{1-r^{2} / R^{2}+A_{2} / r}}
$$

The integral of (39) is difficult. If the third item in radical sign is neglected, we obtain [1].

$$
\begin{aligned}
V^{\prime} & \approx 4 \pi \int_{R_{1}}^{R_{2}} \frac{r^{2} \mathrm{~d} r}{\sqrt{1-r^{2} / R^{2}}}=\left.\frac{4 \pi R^{3}}{3}\left(\arcsin \frac{r}{R}-\frac{r}{R} \sqrt{1-\frac{r^{2}}{R^{2}}}\right)\right|_{R_{1}} ^{R_{2}} \\
& =\frac{4 \pi}{3}\left(R_{2}^{3}-R_{1}^{3}\right)+\frac{2 \pi}{5 R^{2}}\left(R_{2}^{5}-R_{1}^{5}\right)+Q\left(R_{2}, R_{1}, R\right)
\end{aligned}
$$

If we consider factor $A_{2} / r$ in (39), the integral becomes more complex. So in curved space, we have:

$$
M=\frac{4 \pi \rho_{0}}{3}\left(R_{2}^{3}-R_{1}^{3}\right)+\Delta M \neq \frac{4 \pi \rho_{0}}{3}\left(R_{2}^{3}-R_{1}^{3}\right)
$$

Substitute (41) in (37), we get

$$
A_{1}=-\frac{2 G \Delta M}{c^{2}}
$$

Because $R_{1}, R_{2}$ and $\rho_{0}$ can be chosen arbitrarily, we have $\Delta M \neq 0$ and $A_{1} \neq 0$ in general. Therefore, from (35) and (37), we obtain

$$
\begin{gathered}
B_{2}=\frac{\frac{\sqrt{R_{2}-2 G M / c^{2}}}{F\left(R_{2}\right)}-\frac{\sqrt{R_{1}-2 G \Delta M / c^{2}}}{F\left(R_{1}\right)}}{D\left(R_{2}\right)-D\left(R_{1}\right)} \\
C_{2}=\frac{\frac{D\left(R_{1}\right) \sqrt{R_{2}-2 G M / c^{2}}}{F\left(R_{2}\right)}-\frac{D\left(R_{2}\right) \sqrt{R_{1}-2 G \Delta M / c^{2}}}{F\left(R_{1}\right)}}{D\left(R_{1}\right)-D\left(R_{2}\right)}
\end{gathered}
$$

Similarly, because $R_{1}, R_{2}$ and $\rho_{0}$ are arbitrary, we have $B_{2} \neq 0$ and $C_{2} \neq 0$ in general. In this way, all integral constants are determined. In the region $I_{1}$ of spherical cavity, we can write (33) as

$$
g_{00}(r)=\left(1-\frac{2 G \Delta M}{c^{2} r}\right), \quad g_{11}(r)=-\left(1-\frac{2 G \Delta M}{c^{2} r}\right)^{-1}
$$

According to the Newtonian theory, the material distributed outside the spherical cavity with spherical symmetry does not affect the gravity field in the cavity. But according to (45), it will have some effect for the cavity.

\section{The Singularity of the Inner Metric of Hollow Sphere}

According to (45), the metric $g_{00}(r)$ and curvature has singularity at the point $r=0$. This is inherent singularity which can not be eliminated by the coordinate transformation. The seriousness of problem is that for any hollow sphere composed of common material, no matter what are its mass and density, singularity always exists at its center. This does not agree with practical observation. It is impossible actually. On the other hand, we consider 
(14) and let

$$
1-\frac{r^{2}}{R^{2}}+\frac{A_{2}}{r}=0 \quad \text { or } \quad r^{3}-R^{2} r-R^{2} A_{2}=0
$$

It seems that there is a singularity surface beneath two spherical shells. We now discuss this problem. The real number solution of (46) is

$$
\begin{aligned}
r= & {\left[\frac{R^{2} A_{2}}{2}+\sqrt{\left(\frac{R^{2} A_{2}}{2}\right)^{2}-\left(\frac{R^{2}}{3}\right)^{3}}\right]^{\frac{1}{3}} } \\
& +\left[\frac{R^{2} A_{2}}{2}-\sqrt{\left(\frac{R^{2} A_{2}}{2}\right)^{2}-\left(\frac{R^{2}}{3}\right)^{3}}\right]^{\frac{1}{3}}
\end{aligned}
$$

However, if $r$ is really a real number, the following relation should be satisfied

$$
\delta=\left(\frac{R^{2} A_{2}}{2}\right)^{2}-\left(\frac{R^{2}}{3}\right)^{3} \geq 0
$$

Let $r=R_{2}$ in (46) and considering (34), we have

$$
R^{2} A_{2}=-\frac{2 G M R^{2}}{c^{2}}+R_{2}^{3}=R_{1}^{3}-\frac{2 G \Delta M R^{2}}{c^{2}}
$$

In the weak field, the item containing $\Delta M$ can be neglected. By considering (48) and (49), we get

$$
R_{1} \geq\left(\frac{2}{\sqrt{27}}\right)^{\frac{1}{3}}\left(\frac{3 c^{2}}{8 \pi G \rho_{0}}\right)^{\frac{1}{2}}=\frac{9.26 \times 10^{12}}{\sqrt{\rho_{0}}}
$$

We know that even for high density celestial body just as white dwarf, the difference is still very small when we do calculation based on both general relativity and the Newtonian theory. The material density of white dwarf is $\rho_{0}=10^{11} \mathrm{Kg} / \mathrm{m}^{3}$. By using this value in (50), we get $R_{1} \geq 2.7 \times 10^{7} \mathrm{~m}$, similar to the size of white dwarf. For common galaxy, we have $\rho_{0}=10^{-21} \mathrm{Kg} / \mathrm{m}^{3}$. By using this value in (50), we have $R_{1} \geq 3 \times 10^{22} \mathrm{~m}$, which is just the size of galaxies. So (50) can be satisfied for common spheres and (47) becomes

$$
r=\left(\frac{R_{1}^{3}}{2}-\frac{G \Delta M}{c^{2}}+\sqrt{\delta}\right)^{\frac{1}{3}}+\left(\frac{R_{1}^{3}}{2}-\frac{2 G \Delta M}{c^{2}}-\sqrt{\delta}\right)^{\frac{1}{3}}
$$

By developing (51) into the Taylor series, if $\Delta M>0$, we have $r<R_{1}$. That is to say, there is a singularity surface in the cavity. Because the metric of cavity is (33), in stead of (14), there is no singularity surface in the region $R_{1}<r<R_{2}$ inside the hollow sphere. If $\Delta M<0$, we have $r>R_{1}$. In this case, there is a singularity surface in the region $R_{1}<r<R_{2}$.

According to (16), the pressure intensity in the hollow sphere is

$$
\begin{aligned}
p(r) & =B_{2} \mathrm{e}^{-v(r) / 2}-\rho_{0} c^{2} \\
& =\frac{B_{2} \sqrt{r}}{F(r)\left(C_{2}+B_{2} D(r)\right)}-\rho_{0} c^{2}
\end{aligned}
$$

If there is a surface with radius $r=r^{\prime}$ inside the hollow sphere on which we have

$$
C_{2}+B_{2} D\left(r^{\prime}\right)=0
$$

The pressure intensity on the surface will become infinite. Therefore, if there exists black hole in hollow sphere, the black hole would be a spherical surface. Substitute (44) and (45) in (53), we get

$$
D\left(r^{\prime}\right)=\frac{\frac{D\left(R_{2}\right) \sqrt{R_{1}-2 G \rho_{0} \Delta V / c^{2}}}{F\left(R_{1}\right)}-\frac{D\left(R_{1}\right) \sqrt{R_{2}-2 G M / c^{2}}}{F\left(R_{2}\right)}}{\frac{\sqrt{R_{1}-2 G \rho_{0} \Delta V / c^{2}}}{F\left(R_{1}\right)}-\frac{\sqrt{R_{2}-2 G M / c^{2}}}{F\left(R_{2}\right)}}
$$

Because the radii $R_{1}$ and $R_{2}$ are arbitrary, from (54) that we may find a proper $r^{\prime}$ so that (68) can be satisfied. However, on this spherical surface composed of black holes, space-time has no singularity. That is to say, the surface of space-time singularity does not overlap with the surface on which material collapses. This is incomprehensible. It should be noted that up to now we have no any restriction on the mass and density of hollow sphere. This result indicates that common hollow spheres may be unstable. They may collapse into the black hole of spherical surface!

Similarly, because the internal and external radii are arbitrary, let $r=R_{2}$ or $r=R_{1}$, we have $p\left(R_{2}\right) \neq 0$ and $p\left(R_{1}\right) \neq 0$ in general. Because the hollow sphere is placed in vacuum without material outside and inside its two surfaces, this result is also incomprehensible. The singularities of hollow sphere are shown in Figure 1. It is obvious that the results can not be true.

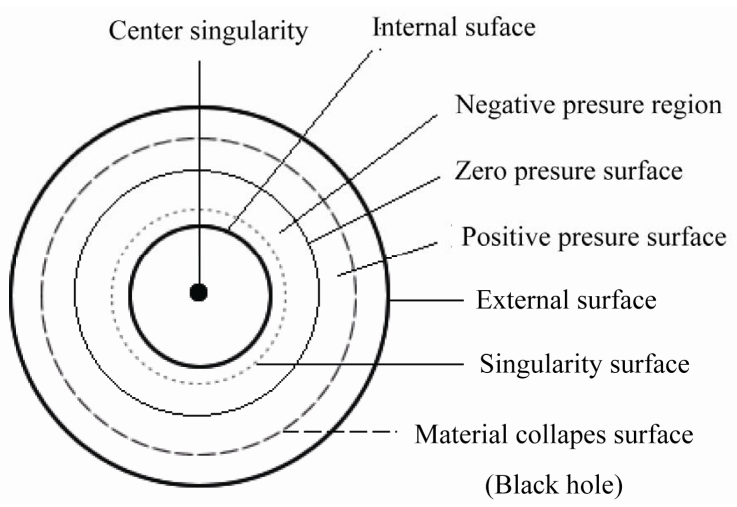

Figure 1. The singularity of hollow sphere. 


\section{The Singularities of Solid Sphere's Metric and Black Holes}

According to the present calculation of general relativity, the internal metric of a common solid sphere has no singularity when the radius of sphere is greater than the Schwarzschild radius. According to the strict calculation in this paper, the situation is completely different. The solid sphere is a special situation of hollow sphere when its internal radius becomes zero. The internal metric of solid sphere is still described by (30), but the conditions of boundary are different. On the spherical surface $r=R_{2}$, we have

$$
\begin{gathered}
1-\frac{2 G M}{c^{2} R_{2}}=1-\frac{R_{2}^{2}}{R^{2}}+\frac{A_{2}}{R_{2}} \\
1-\frac{2 G M}{c^{2} R_{2}}=\frac{F^{2}\left(R_{2}\right)}{R_{2}}\left[C_{2}+B_{2} D\left(R_{2}\right)\right]^{2}
\end{gathered}
$$

In order to determine $A_{2}$ in (55), we have to know the relation between $M$ and $R_{2}$. In curved space, we have

$$
M=\int \frac{\rho_{0} \mathrm{~d} V}{\sqrt{-g_{11}(r)}}=\int_{0}^{R_{2}} \frac{4 \pi \rho_{0} r^{2} \mathrm{~d} r}{\sqrt{1-r^{2} / R^{2}+A_{2} / r}}
$$

By substituting (57) in (55), we can decide $A_{2}$ in principle. We have $A_{2} \neq 0$ in general. If suppose $A_{2}=0$, we have

$$
\begin{aligned}
M & =\rho_{0} \int_{0}^{R_{2}} \frac{4 \pi r^{2} d r}{\sqrt{1-r^{2} / R^{2}}} \\
& =\frac{4 \pi}{3} \rho_{0} R_{2}^{3}+\frac{2 \pi}{5 R^{2}} \rho_{0} R_{2}^{5}+O\left(R_{2}, R\right) \\
& =\frac{4 \pi}{3} \rho_{0} R_{2}^{3}+\Delta M
\end{aligned}
$$

Because $\Delta M \neq 0$, the boundary condition (55) can not be satisfied. We estimate the magnitude of volume's change in curved space based on (58). Let $V^{\prime}=V_{0}+\Delta V$ and omitting high order items, we have

$$
\frac{\Delta V}{V_{0}}=\frac{3 R_{2}^{2}}{10 R^{2}}=\frac{4 \pi G \rho_{0} R_{2}^{2}}{5 c^{2}}
$$

For neutron stars, we have $\rho_{0} \sim 10^{15}$ and $R_{2} \sim 10^{4}$, so $\Delta V / V_{0}=1.8 \times 10^{-4}$. If considering the universe as a uniform sphere, we have $\rho_{0} \sim 10^{-27}$ and $R_{2} \sim 10^{26}$, so $\Delta V / V_{0}=1.8 \times 10^{-2}$. For so-called black hole, we have $R_{2}=c^{2} /\left(3 \pi G \rho_{0}\right)$ according to (4) and $\Delta V / V_{0}=4 / 15=0.27$. For common spheres just as the sum and the earth, $\Delta V / V_{0}$ is a very small but non-zero quantity.

After $A_{2}$ is determined, by substituting it into (28) and (29), we can determine $F^{2}\left(R_{2}\right)$ and $D\left(R_{2}\right)$. However, we can not yet determinate $C_{2}$ and $B_{2}$ only based on (56). Another condition is needed. By considering the fact that the pressure intensity on the surface of sphere should be zero with $p\left(R_{2}\right)=0$, from (16) and (31) we have

$$
\sqrt{R_{2}} B_{2}=\rho_{0} c^{2} F\left(R_{2}\right)\left[C_{2}+B_{2} D\left(R_{2}\right)\right]
$$

From (56) and (60), we obtain

$$
\begin{gathered}
B_{2}=\rho_{0} c^{2} \sqrt{1-\frac{2 G M}{c^{2} R_{2}}} \\
C_{2}=\sqrt{1-\frac{2 G M}{c^{2} R_{2}}}\left[\frac{\sqrt{R_{2}}}{F\left(R_{2}\right)}-\rho_{0} c^{2} D\left(R_{2}\right)\right]
\end{gathered}
$$

Now, all integral constants are determined. The internal metric of solid sphere is

$$
\begin{aligned}
\mathrm{d} s^{2}= & \frac{F^{2}(r)}{r}\left[C_{2}+B_{2} D(r)\right]^{2} \mathrm{~d} t^{2} \\
& -\left(1-\frac{r^{2}}{R^{2}}+\frac{A_{2}}{r}\right)^{-1} \mathrm{~d} r^{2}-r^{2}\left(\mathrm{~d} \theta^{2}+\sin ^{2} \theta \mathrm{d} \phi^{2}\right)
\end{aligned}
$$

Because $F(0) \neq 0$ and $C_{2}+B_{2} D(0) \neq 0$ in general, we have $g_{00}(0) \rightarrow \infty$ and $g_{11}(0) \rightarrow 0$ at point $r=0$, so the infinite of space-time curvature also appears at the center of sphere.

The pressure intensity of solid sphere is also represented by (52), but the integral constants should be represented by (61). In general, we have $F(0) \neq 0$ and $C_{2}+B_{2} D(0) \neq 0$, so we have $p(0)=-\rho_{0}$. That is to say, no mater what are the mass and density of sphere, the pressure intensity at the center of sphere can not be infinite. So-called the singularity black holes in which material collapses towards its center are impossible. Meanwhile, the pressure intensity may become negative value at the center of sphere and its nearby region too. On the other hand, if there is spherical surface with radius $r=r^{\prime}$ so that we have

$$
C_{2}+B_{2} D\left(r^{\prime}\right)=0
$$

the pressure intensity may become infinite on the surface. Substitute (61) in (63), we obtain

$$
D\left(r^{\prime}\right)=D\left(R_{2}\right)-\frac{\sqrt{R_{2}}}{\rho_{0} c^{2} F\left(R_{2}\right)}
$$

Therefore, if black hole exists in solid sphere, it can 


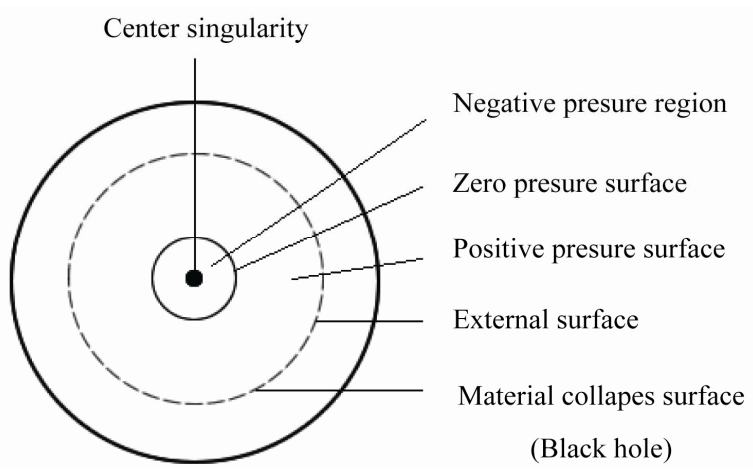

Figure 2. The singularity of solid sphere.

only take the form of a spherical surface. Such solid sphere is not stable for material will collapse to its spherical surface. But according to our common experiences there is no singularity of space-time curvature on the spherical surface. Such result is also uncanny.

The singularity of solid sphere is shown in Figure 2. Notice that we did not impose any restriction for mass and density, and there are so many strange characteristics for common solid spheres. The results are completely different from the current understanding in general relativity. The theory of singularity black hole in the current astrophysics and cosmology has to be reconsidered.

\section{Discussions on the Theorem of Singularity and the Rationality of Current Singularity Black Hole Theory}

S. W. Hawking etc. proved the theorem of singularity by means of the method of differential geometry [4]. The theorem was based on three prerequisite conditions. 1. General relativity was tenable. 2. The law of causality was tenable. 3. There were some points in space-time at which material densities were non-zero. The theorem claimed that if theses three conditions were satisfied, singularity inevitably existed in space-time. Hawking etc. considered singularities as the beginning and ending of time. The Big Bang theory was considered as the beginning of time and the black holes were regarded as the ending of time.

We note that the theorem had no restriction on material's mass and density and did not demand that singularities were embodied in material. That is to say, according to the theorem, singularities may bare in vacuum. In order to avoid this embarrassing situation, Penrose proposed the so-called principle of the universe supervisor. The principle declares that there exists the universe supervisor who prohibit the appearance of bare singularities in vacuum. In other word, due to the existence of the universe supervisors, all singularities will be wrapped in the centers of black holes with great masses and high densities. According to the solutions of the Einstein's equation of gravity, there exist Schwarzschild black holes with spherical symmetry and the Kerr black holes with axial symmetry and so on. The singularities were hidden in the centers of material. In this way, they can not be perceived directly, and physicists seem tolerate their existence.

The results revealed in the paper are consistent with the Hawking theorem of singularity actually. We can consider them as the practical examples of the theorem. By considering the fact that the volume of hollow and solid spheres in curved space are different from that in flat space, the strict calculation reveals that singularities can not be avoided at the centers of common hollow and solid spheres with small masses and low densities. On the other hand, because the pressure intensity can not be infinite at the center of sphere, material can not collapse towards the spherical center. Also the result shows that the pressure intensity may become negative values at the center and its nearby region.

Meanwhile, there may be curved surfaces inside the common hollow and solid spheres on which pressure intensities can become infinite so that material will collapse to them. But the space-time curvatures are still finite on the surfaces. The surfaces of space-time singularity do not always overlap with the surfaces with infinite pressure intensities. All these characters can not agree with our practical experiences of common hollow and solid spheres. They are incomprehensible in physics.

According to the current understanding, the black holes exist at the center of Quasars. However, according to the observations of Rudolf E. Schild and Darryl J. Leiter, the center of Quasar $0957+561$ is a close object, called MECO (Massive Eternally Collapsing Object) [5]. It is not a singularity black hole, and is surrounded by a strong magnetic field. The observation of Rudolf $E$. Schild was consistent with the calculation and analyses in this paper. That is to say, if there are black holes in the universal space, they can only be the Newtonian black holes, not the Einstein's singularity black holes!

More essentially, the true world excludes infinites. A correct theory of physics can not tolerate the existence of infinites, especially singularities in daily life's hollow and solid spheres composed of common material. It is well known that the history of physics is one to overcome infinites. Modern physics grows up in the process to surmount infinites. As revealed in this paper, singularity in general relativity is actually caused by the description method of curved space-time. Physicists and cosmologists should take cautious and incredulous attitude toward the problems of singularity black holes. It is not a scientific attitude to consider singularity black holes as objective existence without any question to them. We 
should think in deep, whether or not our theory has something wrong. When we enjoy the beauty and symmetry of the Einstein's theory of gravity, remember that we should not neglect its limitations and possible mistake.

\section{References}

[1] J. R. Oppenheimer and H. Snyder, "On Continued Gravitational Contraction," Physical Review, Vol. 56, No. 5, 1939, pp. 455-459.

[2] Zhang Yongli, "Introduce to Relativity," The Publishing
Company of Yunnan People, Kunming, 1989, p. 388.

[3] L. B. Feng, X. C. Liu and M. C. Li, "Genarel Relativity," Jilin Science Publishing Company, Jilin, 1995, p. 109.

[4] S. W. Hawking and G. F. R. Eills, "The Large Scale Structure of Space Time," Cambridge University Press, New York, 1972.

[5] R. E. Schild, D. J. Leiter and S. L. Robertson, "Black Hole or Meco: Decided by a thin Luminous Ring Structure Deep within Quasar Q0957 + 561," Journal of Cosmology, Vol. 6, 2010, pp.1400-1437. 